\title{
Identification of genetic loci and candidate genes related to soybean flowering through genome wide association study
}

Minmin Li ${ }^{\dagger}$, Ying Liu ${ }^{\dagger}$, Yahan Tao ${ }^{\dagger}$, Chongjing Xu, Xin Li, Xiaoming Zhang, Yingpeng Han, Xue Yang, Jingzhe Sun, Wenbin Li, Dongmei Li', Xue Zhao and Lin Zhao*

\begin{abstract}
Background: As a photoperiod-sensitive and self-pollinated species, the growth periods traits play important roles in the adaptability and yield of soybean. To examine the genetic architecture of soybean growth periods, we performed a genome-wide association study (GWAS) using a panel of 278 soybean accessions and 34,710 single nucleotide polymorphisms (SNPs) with minor allele frequencies (MAF) higher than 0.04 detected by the specificlocus amplified fragment sequencing (SLAF-seq) with a 6.14-fold average sequencing depth. GWAS was conducted by a compressed mixed linear model (CMLM) involving in both relative kinship and population structure.
\end{abstract}

Results: GWAS revealed that 37 significant SNP peaks associated with soybean flowering time or other growth periods related traits including full bloom, beginning pod, full pod, beginning seed, and full seed in two or more environments at $-\log _{10}(P)>3.75$ or $-\log _{10}(P)>4.44$ were distributed on 14 chromosomes, including chromosome 1, 2, 3, 5, 6, 9, 11, 12, 13, $14,15,17,18,19$. Fourteen SNPs were novel loci and 23 SNPs were located within known QTLs or 75 kb near the known SNPs. Five candidate genes (Glyma.05G101800, Glyma.11G140100, Glyma.11G142900, Glyma.19G099700, Glyma.19G100900) in a 90 kb genomic region of each side of four significant SNPs (Gm5_27111367, Gm11_10629613, Gm11_10950924, Gm19_34768458) based on the average LD decay were homologs of Arabidopsis flowering time genes of AT5G48385.1, AT3G46510.1, AT5G59780.3, AT1G28050.1, and AT3G26790.1. These genes encoding FRI (FRIGIDA), PUB13 (plant U-box 13), MYB59, CONSTANS, and FUS3 proteins respectively might play important roles in controlling soybean growth periods.

Conclusions: This study identified putative SNP markers associated with soybean growth period traits, which could be used for the marker-assisted selection of soybean growth period traits. Furthermore, the possible candidate genes involved in the control of soybean flowering time were predicted.

Keywords: Genome wide association study, Candidate genes, Soybean growth periods, Genetic improvement

\section{Background}

Soybean (Glycine max) is a major crop of agronomic importance grown across a wide range of latitudes from $50^{\circ} \mathrm{N}$ to $35^{\circ} \mathrm{S}$ [1]. However, soybean varieties are limited to narrow latitudes due to the photoperiod sensitivity. The complex growth period traits are controlled by both internal and external factors, which make great effects on crop adaptability, biomass and economic yield [2]. As a

\footnotetext{
*Correspondence: yy841026@163.com; xuezhao@neau.edu.cn; zhaolinneau@126.com

${ }^{+}$Minmin Li, Ying Liu and Yahan Tao contributed equally to this work. Key Laboratory of Soybean Biology of Ministry of Education, China (Key Laboratory of Biology and Genetics \& Breeding for Soybean in Northeast China), Northeast Agricultural University, Harbin, China
}

typical photoperiod-sensitive short-day plant, soybean photoperiod is the main climatic factor that determines its growth periods and adaptability to different ecological zones. The genetic mechanisms of soybean flowering time and maturity were complex [3]. Previous studies identified at least 11 major-effect loci affecting flowering and maturity of soybean, which were designated as $E 1$ to $E 10$ [4-14], and the $J$ locus for "long juvenile period" [15], which was important for soybean to adapt to high latitude environments. E1,E2, E3, E4,E9 and $J$ had been cloned or identified. Of these, E1 encoding a nuclear-localized B3 domain-containing protein was induced by long days. E2

(c) The Author(s). 2019 Open Access This article is distributed under the terms of the Creative Commons Attribution 4.0 International License (http://creativecommons.org/licenses/by/4.0/), which permits unrestricted use, distribution, and 
encoded a homolog of GIGANTEA and controlled soybean flowering time by regulating GmFT2a [1]. E3 and E4 encoded phytochrome PHYA3 and PHYA2 proteins [7, 16]. $J$ was the dominant functional allele of GmELF3 [17]. In addition to these major loci, many minor-effect quantitative traits loci (QTLs) related to soybean flowering time and maturity had also been identified. To date, at least 104,6 , 5, and 5 QTLs associated with first flower, pod beginning, seed beginning, and seed fill had been reported in soybean (SoyBase, www.soobbase.org), respectively. Many other orthologs of Arabidopsis flowering genes such as GmCOLs [18], GmSOC1 [19], and GmCRY [20] had also been identified. Taken together, these results showed a complex genetic basis of flowering and maturity in soybean.

Genome-wide association study (GWAS), based on linkage disequilibrium (LD), had emerged as a powerful tool for gene mapping in plants to take advantage of phenotypic variation and historical recombination in natural populations and overcome the limitations of biparental populations, resulting in higher QTL mapping resolution [21-23]. So far, the next-generation sequencing technologies such as genotyping by sequencing (GBS), restriction site-associated DNA sequencing (RAD-seq) and specific-locus amplified fragment sequencing (SLAF-seq) had been used to detect highquality single nucleotide polymorphisms (SNPs) for GWAS in soybean [24-26]. The Illumina Infinium SoySNP50K BeadChip was used to genotype the population consisting of 309 early-maturing soybean germplasm resources, and ten candidate genes homologous to Arabidopsis flowering genes were identified near the peak SNPs associated with flowering time detected via GWAS [3]. Ninety-one soybean cultivars of maturity groups (MGs) 000-VIII were subjected to GWAS using Illumina SoySNP6K iSelectBeadChip, and 87 SNP loci associated with soybean flowering were identified [27]. Eight hundred and nine soybean cultivars were sequenced on Illumina HiSeq 2000 and 2500 sequencer, GWAS identified 245 significant genetic loci associated with 84 agronomic traits by single and multiple marker frequentist test (EMMAX), 95 of which interacted with other loci [28]. The recombinant inbred line (RIL) population were genotyped by RAD-seq in 2 year studies, the high-density soybean genetic map was constructed and 60 QTLs that influenced six yield-related and two quality traits were identified [29]. SLAF-seq technology had several obvious advantages, such as high throughput, high accuracy, low cost and short cycle, and this technology had been reported in haplotype mapping, genetic mapping, linkage mapping and polymorphism mapping. It could also provide important bases for molecular breeding, system evolution and germplasm resource identification. A total of 200 diverse soybean accessions with different resistance to SCN HG Type 2.5.7 were genotyped by SLAF-seq for GWAS, and the results revealed 13 SNPs associated with resistance to SCN HG Type 2.5.7, and 30 candidate genes underlying SCN resistance were identified [30]. In the present study, we performed GWAS for soybean growth period traits in the total of 278 soybean accessions genotyped by SLAFseq and identified 37 significantly associated SNPs in two or more environments and five potential candidate genes regulating growth periods. Our studies provided an insight into the genetic architecture of soybean growth periods and the identified candidate markers and genes would be valuable for the marker-assisted selection of soybean.

\section{Results}

Phenotype statistics of $\mathbf{2 7 8}$ soybean germplasms

Field experiments were conducted in three different locations (Harbin, Changchun, Shenyang) in China for 2 years (2015 and 2016). The statistical analysis on the results of phenotype indicated that six growth period characteristics including flowering time, full bloom, beginning pod, full pod, beginning seed, and full seed of 278 soybean germplasms (Fig. 1, Additional file 1) showed abundant phenotypic variation (14.9 43.6\%) (Additional file 2), and reflected their great potential of genetic improvement. After normalizing, the six growth period characters of 278 soybean germplasms above showed normal distributions without any significant skewness, which could be used for the subsequent statistical analysis (Additional file 10: Figure S1). Correlation analysis showed that there were high correlations between flowering time and full bloom (0.90 0.98), beginning pod (0.96 0.88), full pod (0.87 0.94), beginning seed (0.84 0.93), and full seed (0.83 0.90) (Additional file 11: Figure S2), implying that the flowering time and the other five growth periods in soybean might be controlled by the same genetic factors.

The results of ANOVA showed that the heritability of flowering time, full bloom, beginning pod, full pod, beginning seed, and full seed in soybean were quite high (94.7 96.2\%) (Additional file 3), indicating that the growth periods traits were mainly significantly affected by genetic variability. Therefore, the probability of obtaining the off springs with excellent target traits was large by selecting them in the early generation of breeding using a strict criteria [32]. However, the flowering time, full bloom, beginning pod, full pod, beginning seed, and full seed in soybean were also affected by environmental factors such as geographical location and year, as well as environment-genotype interactions $(P<$ 0.01 ) (Additional file 3), which made the majority of soybean bloom the earliest in Shenyang (lower latitude), whereas bloom the latest in Harbin (higher latitude) in 


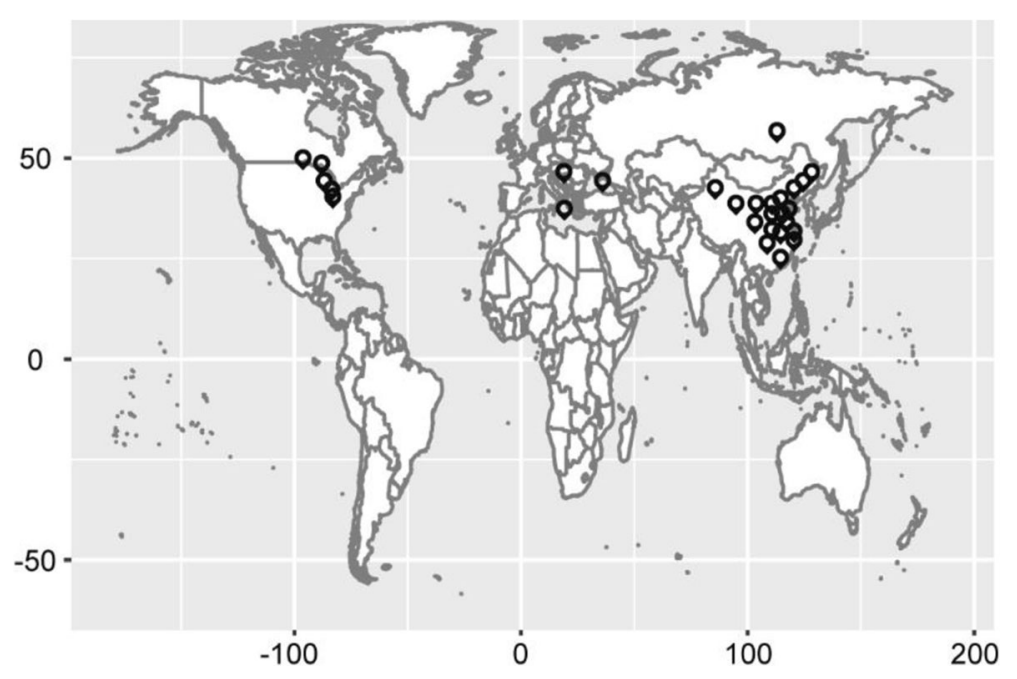

Fig. 1 Geographical distribution of 278 soybean germplasm resources. The map was made by the completely free software R [31] version 3.6.1 (https://mirrors.tuna.tsinghua.edu.cn/CRAN/)

the same year (Additional file 1, Additional file 2). Fortyone soybean germplasms flowering earlier (27.5 38.5 d) and 53 flowering later (58 113 d) with stable performance (Additional file 4) were screened by GGE biplot in six environments to avoid the impact of the environment, which could be considered for broadening the genetic basis for the improvement of soybean germplasms to produce greater super-parent effects.

\section{Linkage disequilibrium (LD), population structure and kinship analyses}

The DNA sequencing data had been uploaded [33]. The dataset of 34,710 SNPs with MAF higher than 0.04 covering all 20 chromosomes was used to conduct GWAS (Additional file 5, Additional file 12: Figure S3). The largest number of SNPs was identified on chromosome 18 (2708 SNPs) followed by chromosome 15 (2515 SNPs), and the smallest of SNPs was found on chromosomes 11 (961 SNPs) and chromosomes 12 (1079 SNPs) (Additional file 6, Fig. 2). The highest marker density was detected on chromosome 15 (one SNP per $20.58 \mathrm{~kb}$ ), and the smallest one was identified on chromosome 12 (one SNP per $37.15 \mathrm{~kb}$ ), while the average marker density was approximately one SNP per $28.36 \mathrm{~kb}$ (Additional file 6). It was found that the average LD decay distance of the population was about $300 \mathrm{~kb}\left(\mathrm{r}^{2}=0.5\right)$ by 34,710 SNP markers for LD analysis (Fig. 3a). Previous studies had shown that the LD decay distance of soybean natural population was $250 \sim 375 \mathrm{~kb}$ [34], which was similar to the results of this study, indicating that the marker coverage obtained in this study was high enough for GWAS. The population structure of 278 soybean accessions obtained by principal component analysis of 34,710 SNPs reflected the subgroup structure (Fig. $3 \mathrm{~b}$ and c), suggesting that geographic isolation was important for
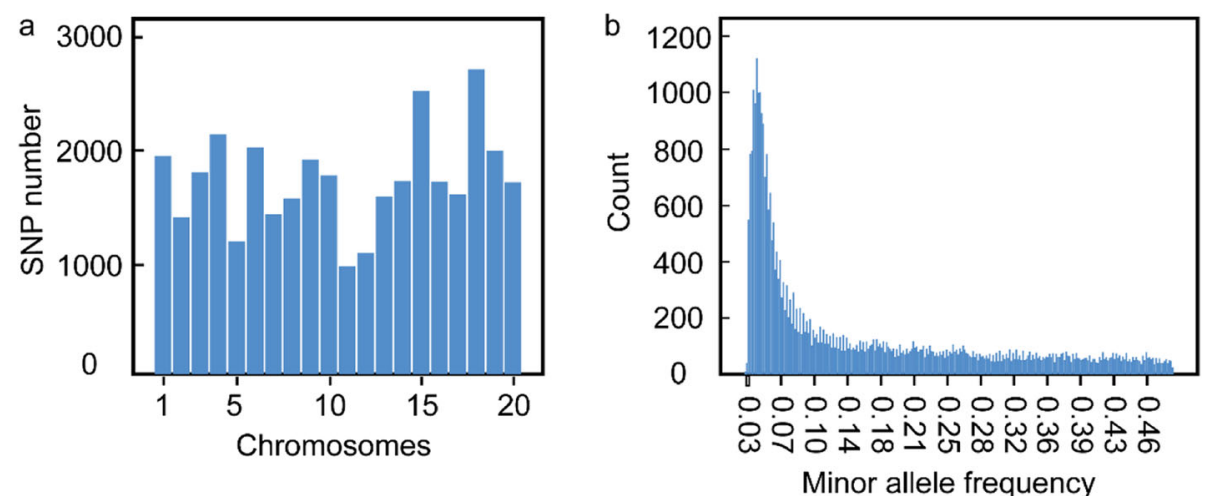

Fig. 2 Single-nucleotide polymorphism for 278 soybean accessions. a Distribution of the SNP markers across 20 soybean chromosomes. b Minor allele frequency distribution of SNP alleles 


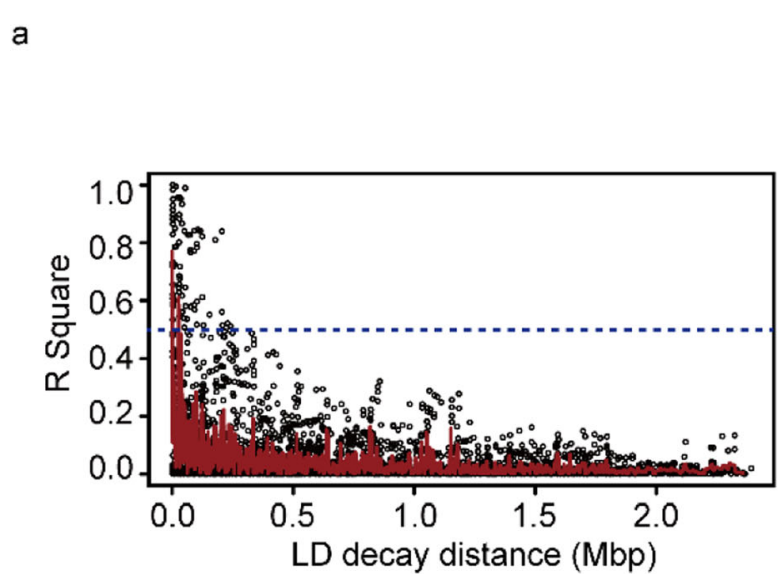

C

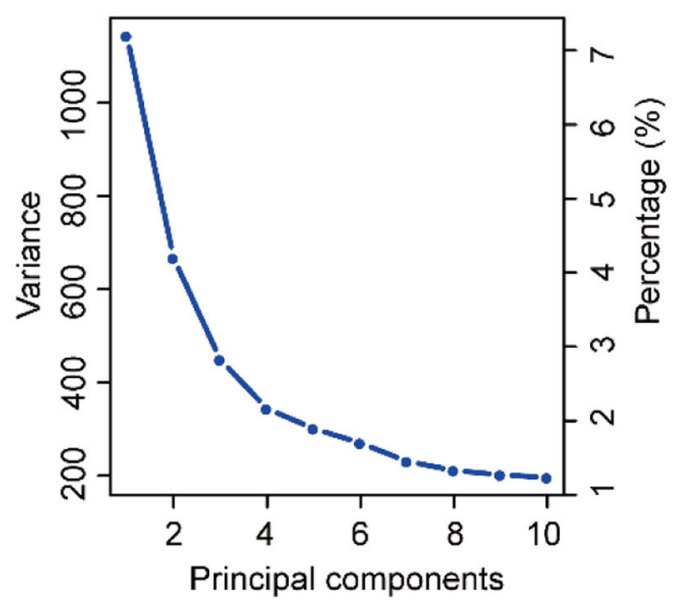

b

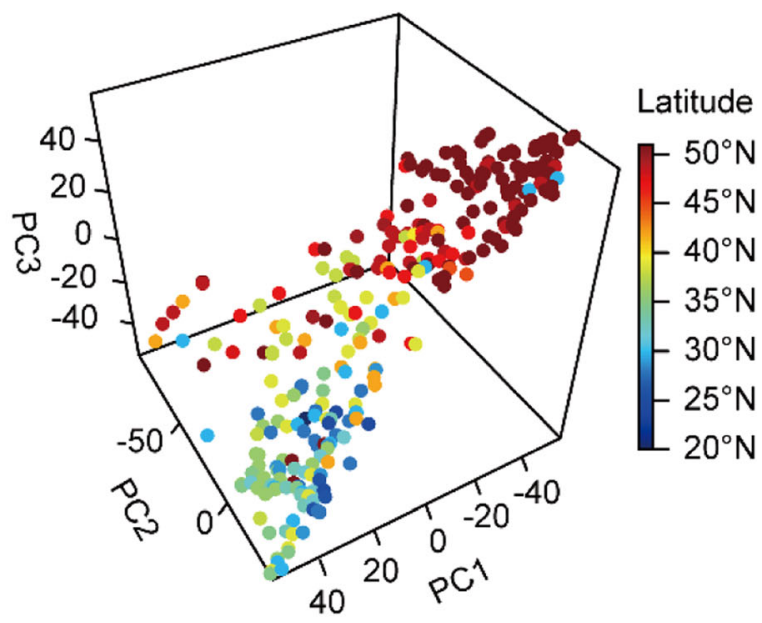

d

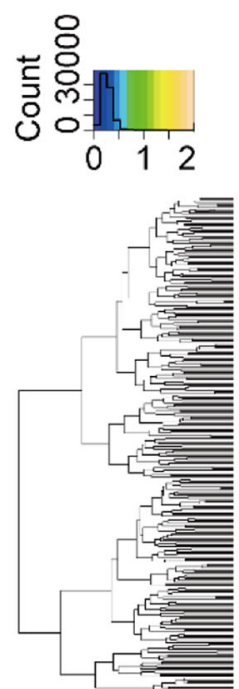

Fig. 3 The linkage disequilibrium (LD), principal component and kinship analyses of soybean genetic data. a The estimated average linkage disequilibrium (LD) decay of soybean genome. The dashed line in blue indicated the position where $r^{2}$ was 0.5 . b The first three principal components of 34,710 SNPs used in the GWAS indicated little population structure among 278 tested accessions. c The population structure of the soybean germplasm collection reflected by principal components. $\mathbf{d}$ The heat map of the kinship matrix of the 278 soybean accessions calculated from the same 34,710 SNPs used in the GWAS, suggesting low levels of relatedness among 278 individuals

shaping genetic differentiation of soybean. The kinship matrix among 278 soybean accessions calculated based on 34,710 SNPs indicated a lower level of genetic relatedness among soybean individuals (Fig. 3d).

\section{Identification of genetic loci and candidate genes through GWAS}

The CMLM-PCA + K statistical model considering the covariates composed of population structure and kinship matrix was used for GWAS to prevent false positivity [35]. The total of 223 SNP loci associated with flowering time, full bloom, beginning pod, full pod, beginning seed, and full seed in one or more environments were all considered to be candidate sites for flowering time in soybean, because the correlation analysis above demonstrated that these six growth period traits may be controlled by the same genetic factors (Fig. 4, Additional file 7, Additional file 8). Among them, 186 SNPs detected in one environment may be susceptible to environmental influences, 37 SNPs that could explain $17.41 \sim 21.95 \%$ phenotypic variation in two or more environments could be stably inherited in different environments, and it was considered that there would be key genes controlling flowering time nearby.

Twenty-three of 37 SNPs were located within the known QTLs or located $75 \mathrm{~kb}$ near the known SNPs controlling soybean growth periods, indicating the feasibility of the natural population for GWAS (Additional file 8). In addition, 14 unreported SNPs (Gm1_1929268, Gm1_55250122, 


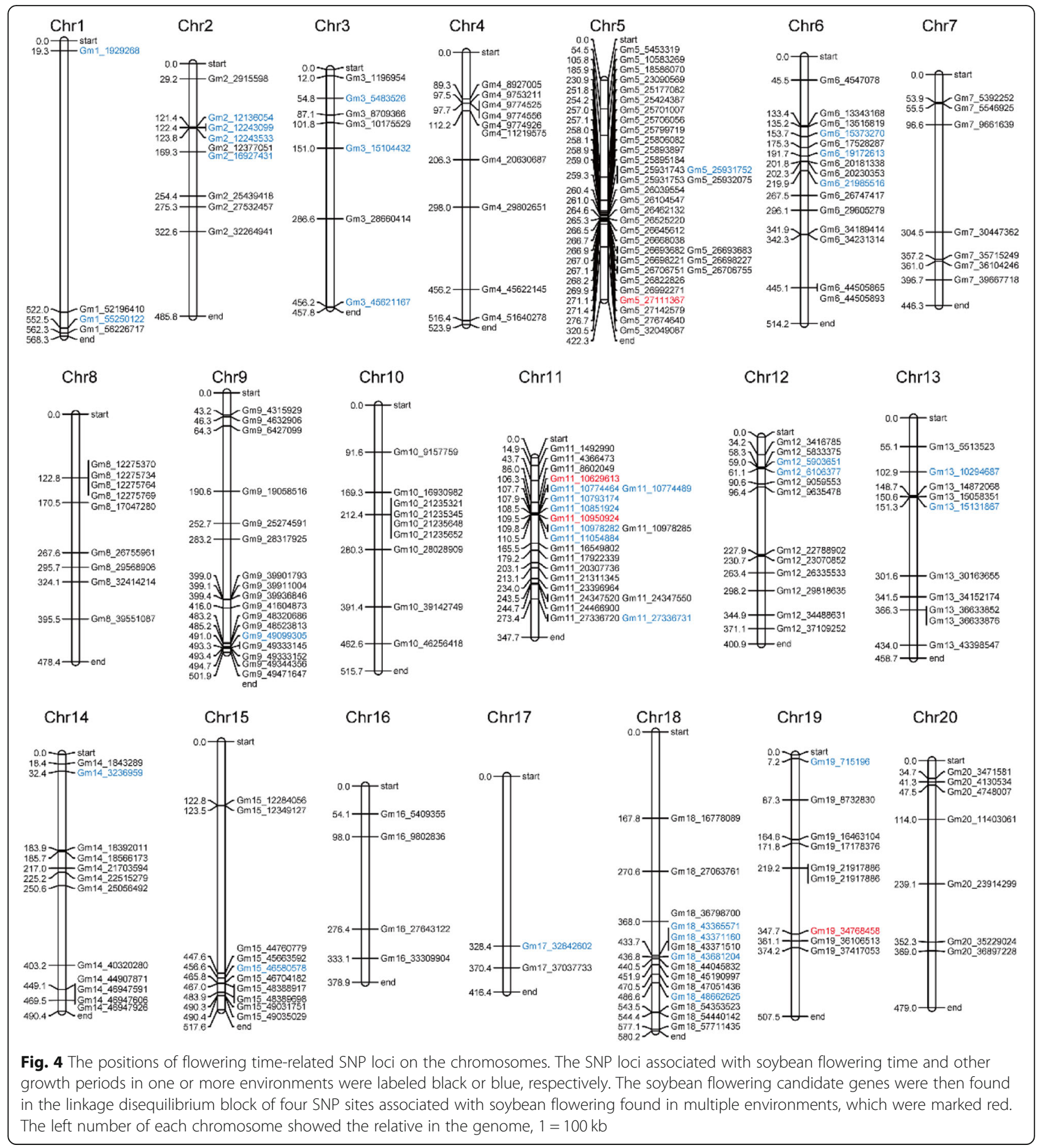

Gm2_12136054, Gm2_12243533, Gm3_15104432, Gm3 45621167, Gm5_27111367, Gm9_49099305, Gm12_61063 77, Gm14_3236959, Gm15_46580578, Gm17_32842602, Gm19_715196, Gm19_34768458) that may control soybean flowering were found on ten chromosomes 1, 2, 3, 5, 9, 12, 14, 15, 17 and 19. A total of 291 genes (Additional file 9) within the linkage disequilibrium (LD) block $\left(r^{2}>0.5\right)$ of 37 significant SNPs were screened, and we further predicted five homologs (Glyma.05G101800, Glyma.11G140100, Glyma.11G142900, Glyma.19G099700, Glyma.19G100900) (Table 1) of flowering time genes of AT5G48385.1, AT 3G46510.1, AT5G59780.3, AT1G28050.1, and AT3G26 790.1 in Arabidopsis that played important roles in flowering pathway as candidate genes related to soybean flowering time within the $90 \mathrm{~kb}$ genomic region of four significant SNPs (Gm5_27111367, Gm11_10629613, Gm11_10950924, 
Table 1 Five candidate genes related to soybean flowering time

\begin{tabular}{lllll}
\hline Candidate Genes & Locus & Annotation & $\begin{array}{l}\text { Distance from a gene to SNP } \\
(\mathrm{kb})\end{array}$ & Functional description \\
\hline Glyma.05G101800 & Gm5_27111367 & AT5G48385.1 & -47.91 & FRIGIDA-like protein \\
Glyma.11G140100 & Gm11_- & AT3G46510.1 & +47.56 & plant U-box 13 \\
& 10629613 & AT5G59780.3 & -35.11 & Transcription factor MYB59-related \\
Glyma.11G142900 & Gm11_- & 10950924 & AT1G28050.1 -85.90 & $\begin{array}{l}\text { Zinc finger protein CONSTANS-LIKE 14-related transcription } \\
\text { factor }\end{array}$ \\
Glyma.19G099700 & Gm19_ & 34768458 & B3 domain-containing transcription factor FUS3 \\
\hline
\end{tabular}

If the candidate gene is located upstream of the SNP, the distance from the gene to the SNP is indicated by a negative sign. Instead, it is represented by a positive sign

Gm19_34768458) (Fig. 5). Glyma.05G101800 encoding FRIGIDA-like protein was located at $47.91 \mathrm{~kb}$ upstream of Gm5_27111367, and 251 soybeans with major allele G at this locus flowered 23.82, 19.33, 34.94, 19.03, and 32.07 days earlier than the 27 soybeans with minor allele $\mathrm{T}$ in five environments of 2015 Harbin, 2015 Changchun, 2016 Changchun, 2015 Shenyang, 2016 Shenyang, respectively (Fig. 6). Glyma.11G140100 encoding PUB13 (plant U-box 13) protein was located at $47.56 \mathrm{~kb}$ downstream of $\mathrm{Gm} 11_{-}$ 10629613, and 253 soybeans carrying major allele $G$ at this locus flowered 28.23, 22.01, 37.48, 22.72, and 33.90 days earlier than the 25 soybeans with minor allele A in 2015 Harbin, 2015 Changchun, 2016 Changchun, 2015 Shenyang, 2016 Shenyang, respectively (Fig. 6). Glyma.11G142900 encoding MYB59 protein was located at $35.11 \mathrm{~kb}$ upstream of Gm11_10950924, and 251 soybeans with major allele $\mathrm{G}$ at this locus flowered 33.51, 29.13, 44.52, 26.27, and 39.73 days earlier than the 27 soybeans with minor allele A in 2015 Harbin, 2015 Changchun, 2016 Changchun, 2015 Shenyang, 2016 Shenyang, respectively (Fig. 6). Glyma.19G099700 and Glyma.19G100900 encoding CONSTANS and FUS3 proteins were located at 85.90 and $37.60 \mathrm{~kb}$ downstream of Gm19_34768458, respectively, and 238 soybeans with the major frequency allele $\mathrm{T}$ at this locus flowered 7.68, 9.21, 5.72, 6.10, and 7.56 days earlier than the 40 soybeans with the alternative allele A in 2015 Harbin, 2015 Changchun, 2016 Changchun, 2015 Shenyang, 2016 Shenyang, respectively (Fig. 6). The other growth periods also showed the similar tendency with the first flowering time between two alleles of each associated SNP marker (Fig. 6). These four markers Gm5_27111367, Gm11_10629613, Gm11_10950924, and Gm19_34768458 could be targets for breeders for marker assisted selection of soybean growth periods traits.

\section{Discussion}

Six soybean growth periods were significantly affected by genetic-environment interaction

Soybean is a short-day plant with induced cumulative effects by short days, and the flowering time of soybeans and other growth periods were quantitative traits controlled by multiple genes. The six growth periods (flowering time, full bloom, beginning pod, full pod, beginning seed, and full seed) of 278 soybean germplasm resources in this study were highly variable (14.9 43.6\%) in different environments, indicating that the natural population could be used for the genetic improvement of growth periods. The high heritability (94.7 96.2\%) of six growth periods indicated that they were mainly affected by genetic factors. In addition, soybean growth periods were significantly or extremely significantly affected by environmental and genotype-environment interaction, indicating that in addition to genetic effects, photoperiod and temperature conditions in different planting environments played crucial roles in determining the growth periods, which directly determined whether soybeans grown in different ecological environments could flower and mature normally. The growth periods of soybean determined the latitude range suitable for planting, so it was of great significance to study the characteristics of soybean growth periods. In this study, the genetic relationship among 94 stable soybean germplasms, including 41 earlier and 53 later flowering soybean varieties screened by GGE was far from each other, which could be qualified as hybrid breeding parent [36].

\section{The LD decay rate of soybean was higher than cross- pollinated species due to genetic bottleneck}

Increased LD was a hallmark of genetic bottlenecks, the greater LD decay rate for self-pollination was expected to be higher than that of cross-pollinated species [37]. As the physical distance increases, the LD decay of the entire genome was estimated to be decayed to $r^{2}=0.5$ within approximately $300 \mathrm{~kb}$, consistent with previous studies in soybean $(250 \sim 375 \mathrm{~kb})$ [34], similar to the other selfpollinated species such as rice $(123 \sim 167 \mathrm{~kb})$ and sorghum $(150 \mathrm{~kb})[38,39]$, but much larger than the crosspollinated species such as maize (1 10 kb) [40]. The lower density of SNPs was suitable for GWAS in soybean as 


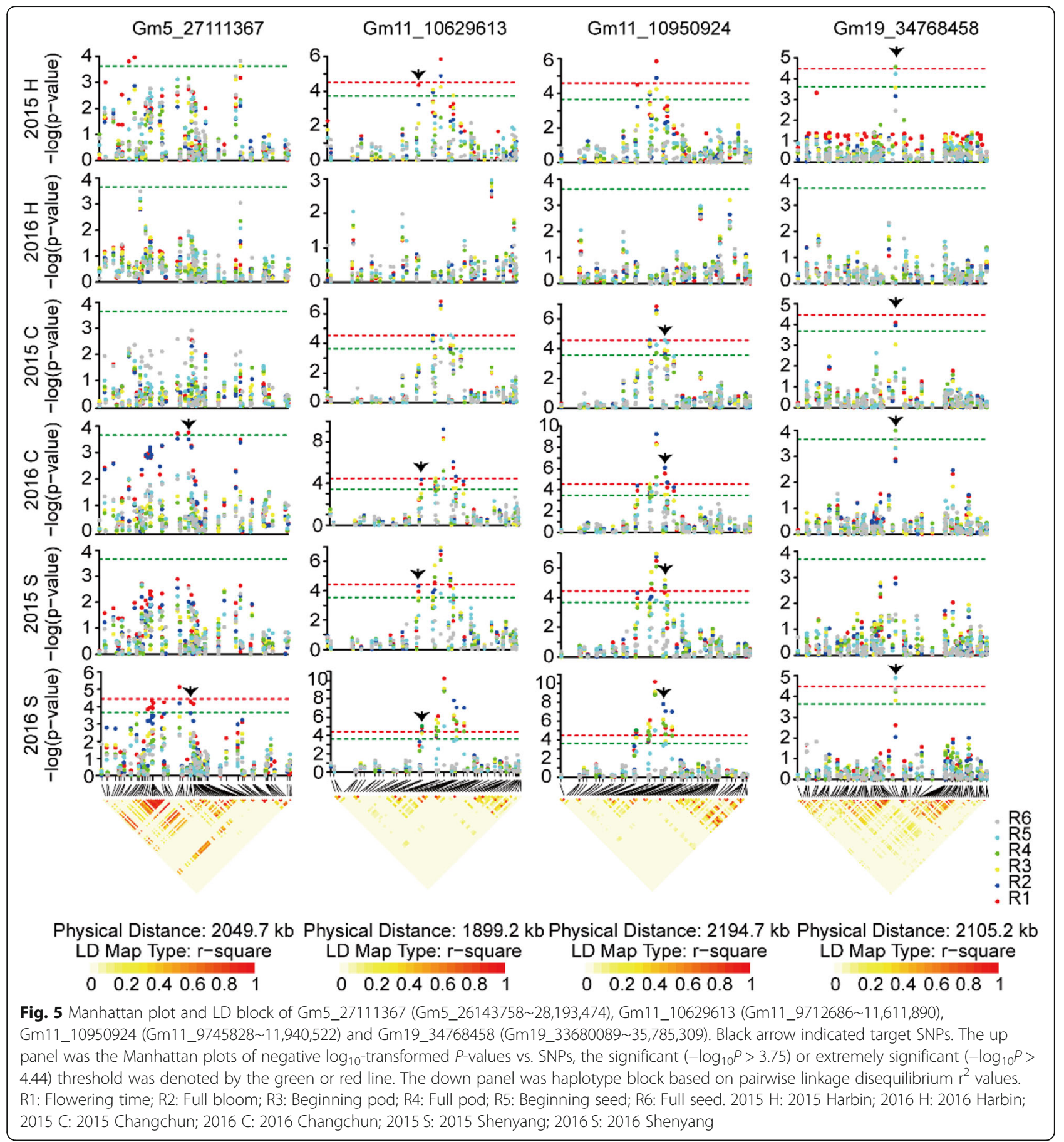

compared with other crops like rice, sorghum and maize, therefore, LD decay rate was the primary factor limiting the mapping resolution in GWAS for soybean.

\section{Determination of 23 known and 14 new soybean flowering time loci}

To date, a number of QTLs associated with soybean growth periods had been reported. In the present study, a total of 37 SNPs distributed on ten chromosomes (chromosomes 1, 2, 3, 5, 9, 12, 14, 15, 17 and 19) were associated with soybean flowering time or the other growth periods in two or more environments. Among the 37 environmental stable association signals, 23 SNPs were overlapped with known QTL or located $75 \mathrm{~kb}$ near the known SNPs controlling soybean growth periods. For instance, two SNPs, Gm2_12243099 and Gm3_5483526, were identified at 73.01 and $18.97 \mathrm{~kb}$ near Gm2_12316110 [28] and Gm03_5502496 [27], respectively. All the four SNPs, 


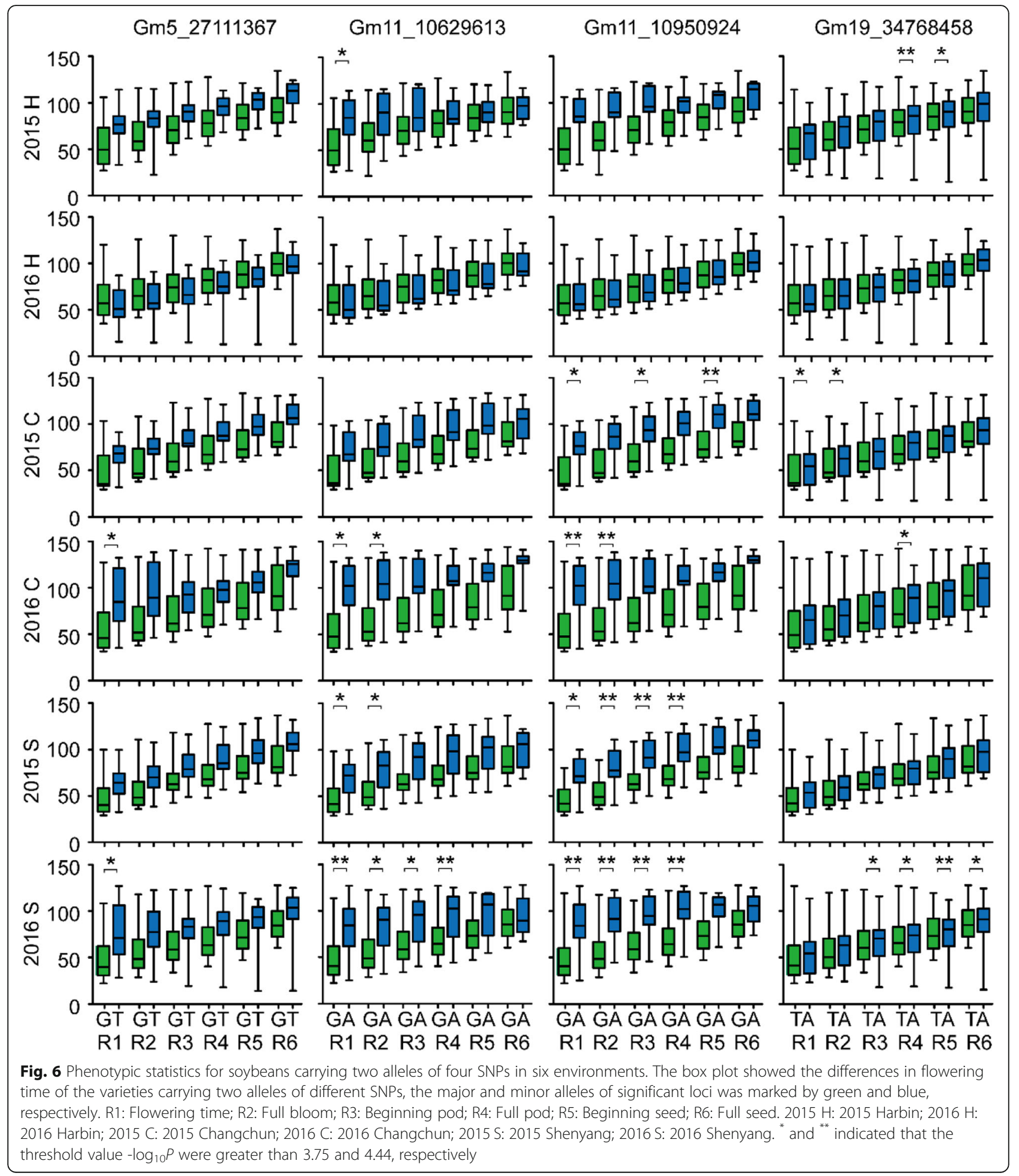

Gm11_10774464, Gm11_10774489, Gm11_10793174, and Gm11_10851924 were identified near Gm11_10847172 [28]. In addition, 14 new SNPs were identified to be significantly different from the major QTLs or reported SNPs and the molecular mechanisms of these new loci needed to be further studied.
Five candidate genes were identified in different flowering pathways

The regulation of flowering time is a very complicated process that is influenced by both genetic factors and external environmental factors. The precise control of flowering time is achieved by the combination of 
various signals generated by these two aspects [41]. To date, approximately six genetic pathways for the promotion or repression of flowering time have been identified in Arabidopsis, including photoperiod, temperature, vernalization, gibberellin (GA) biosynthesis, autonomous and aging pathways [42]. In photoperiod pathway, Arabidopsis thaliana is a genetic model system for photoperiodic responses in plants, and flowers earlier in long days than in short days. In the present study, five homologous genes (Glyma.05G101800, Glyma.11G140100, Glyma.11G142900, Glyma.19G099700, Glyma.19G100900) of Arabidopsis flowering genes of AT5G48385.1, AT3G46510.1, AT5G 59780.3, AT1G28050.1, and AT3G26790.1 that played important roles in Arabidopsis flowering pathway were identified to probably participate in the regulation of soybean growth periods based on strong correlation peak SNP and LD block of four significant SNPs (Gm5 27111367, Gm11_10629613, Gm11_10950924, Gm19_ 34768458). The Arabidopsis transcription factor CONSTANS (CO) played a central role in promoting flowering in LDs. CO protein directly binded to the motifs in the proximal promoter of its major target gene $F L O W-$ ERING LOCUS T (FT) to promote flowering [43, 44]. After transcriptional activation by $C O$, FT protein moved to the shoot apex, where it induced transcriptional reprogramming of the meristem to form an inflorescence meristem and subsequently flowered [45]. Thus, Glyma.19G099700, located at $85.90 \mathrm{~kb}$ downstream of Gm19_34768458, and encoding a ZINC FINGER PROTEIN CONSTANS-LIKE 14-RELATED transcription factor might be involved in soybean photoperiod control of flowering pathway. In vernalization pathway, FRI (FRIGIDA) was located upstream of FLC (FLOWERING LOCUS C) and regulated vernalization by regulating FLC [42]. The two helix-helical domains of FRI protein interacted with the nucleus cap-binding complex $(\mathrm{CBC})$ to increase transcription and efficient splicing of the flowering inhibitor FLC and delayed flowering [46]. Then, a FRIGIDA-like protein encoded by Glyma.05G101800 was located at $47.91 \mathrm{~kb}$ upstream of Gm5_27111367, but there was no vernalization in soybean, and the function of soybean FRI needed to be validated whether it was related to flowering time. PUB13 was a negative regulator of flowering time under middle- and long-day conditions in which the expression of SOC1 (SUPPRESSOR OF OVEREXPRESSION OF CONSTANS1) and $F T$ was induced while $F L C$ expression was suppressed in Arabidopsis [47]. Glyma.11G140100 located at $47.56 \mathrm{~kb}$ upstream of Gm11_ 10629613 might be considered to be associated with soybean flowering. MYB59 was induced during the light-todark transition [48] and was regulated by the circadian cycle with peak expression in the evening, probably due to its regulation by CIRCADIAN CLOCK ASSOCIATED 1
(CCA1) which was expressed in the morning [49]. CCA1 acted as a transcriptional repressor by associating to the ELF4 promoter [50] and the ELF3 promoter [51] of the photoperiodic flowering pathway. Therefore, the transcription factor MYB59-related Glyma.11G142900 located at $35.11 \mathrm{~kb}$ upstream of Gm11_10950924 might be considered to be associated with soybean flowering. Glyma.19G100900 located at $37.60 \mathrm{~kb}$ downstream of Gm19 34768458 was a B3 domain-containing transcription factor FUS3, which was also identified as a candidate gene of soybean flowering time. Arabidopsis plants overexpressing FUS3 post-embryonically in the L1 layer (ML1p:FUS3) showed late flowering and other developmental phenotypes [52].

\section{Conclusions}

GWAS was powerful in dissecting complex traits and identifying candidate genes. Fourteen novel SNPs and 23 SNPs that located within known QTLs or $75 \mathrm{~kb}$ near the known SNPs associated with soybean flowering time or other related growth period traits in this study may have great potential for soybean yield improvement. Five candidate genes related to soybean flowering time might serve as promising targets for studies of molecular mechanisms underlying growth period traits in soybean.

\section{Methods \\ Plant materials and phenotypic evaluation}

The soybean growth period traits including flowering time, full bloom, beginning pod, full pod, beginning seed, and full seed of the natural population consisting of 278 diverse soybean accessions with varied maturity and growth habit characteristics were recorded and used for GWAS. Two hundred sixty-four and fourteen soybean germplasms [53] were from China and other countries $\left(20^{\circ} 13^{\prime} \mathrm{N} \sim 61.5^{\circ} \mathrm{N}\right)$ (Fig. 1, Additional file 1). The map was completed by 'ggplots', 'colorspace', 'ggmap', 'sp', 'maptools', 'maps', and 'labeling' packages in R software $[54,55]$. All the soybean germplasms were sowed in experimental farms in three different latitudes of Harbin $\left(45^{\circ} 75^{\prime} \mathrm{N}, 126^{\circ} 63^{\prime} \mathrm{E}\right)$, Changchun $\left(43^{\circ} 88^{\prime} \mathrm{N}, 125^{\circ} 35^{\prime} \mathrm{E}\right)$ and Shenyang $\left(41^{\circ} 44^{\prime} \mathrm{N}, 123^{\circ} 30^{\prime} \mathrm{E}\right)$ in China using randomized complete block designs with three replicates in 2015 and 2016. Each experimental block consisted of 2 $\mathrm{m}$ long rows with $0.6 \mathrm{~m}$ row spacing and $0.05 \mathrm{~m}$ plant spacing. The soybean emergence stages and the reproductive periods such as flowering time, full bloom, beginning pod, full pod, beginning seed, and full seed were recorded as described by Fehr and Caviness [56], and the days from emergence to reproductive periods were calculated. Each stage was defined to have occurred if at least $50 \%$ of the individual plants of a given soybean variety had reached that stage. 


\section{Statistical analysis}

The descriptively statistical analyses were carried out by SPSS19.0 [57]. The phenotypic data of flowering time, full bloom, beginning pod, full pod, beginning seed, and full seed for 278 soybean germplasms were ranked by individual cases and transformed into variables obeying standard normal distribution using SPSS19.0 [57] for correlation analysis, variance analysis (ANOVA) and GGE biplot. Correlation analysis of flowering time and full bloom, beginning pod, full pod, beginning seed, full seed in six environments were identified using Pearson's correlation coefficients of "Performance Analytics" package in R software [31]. ANOVA was performed following the standard procedure of a mixed effect model by DPS v14.1.0 to determine the level of the significance of genotype differences, locations, cultivation years and their interactions [58]. Genotype and location were considered as fixed effects, while year was considered as a random effect. The phenotypic observation $Z_{i j k r}$ was modeled as:

$$
\begin{aligned}
Z_{i j k r}= & \mu+G_{i}+L_{j}+Y_{k}+B_{r}\left(L_{j} Y_{k}\right)+G L_{i j}+G Y_{i k} \\
& +L Y_{j k}+G L Y_{i j k}+e_{i j k r}
\end{aligned}
$$

where $Z_{i j k r}$ was the response variable; $\mu$ was the grand mean; $G_{i}$ was the genotype effect; $L_{j}$ was the location effect; $Y_{k}$ was the year effect; $B_{r}\left(L_{j} Y_{k}\right)$ was the block effect; $G L_{i j}$ was the genotype-by-location interaction; $G Y_{i k}$ was the genotype-by-year interaction; $L Y_{j k}$ was the location-by-year interaction; $G L Y_{i j k}$ was the genotype-by-location-by year interaction and $e_{i j k r}$ was the residual error. These components were used to calculate broad-sense heritability $\left(h^{2}\right)$ for soybean flowering time, full bloom, beginning pod, full pod, beginning seed, and full seed, the calculation is based on the following formula [59]:

$$
h^{2}=\frac{\sigma_{g}^{2}}{\sigma_{g}^{2}+\sigma_{g y}^{2} / y+\sigma_{g l}^{2} / l+\sigma_{g l y}{ }^{2} / l y+\sigma_{\varepsilon}^{2} / r l y}
$$

where $\sigma_{g}{ }^{2}$ was genotype, $\sigma_{g l}{ }^{2}$ was genotype-by-location, $\sigma_{g y}{ }^{2}$ was genotype-by-crop year, $\sigma_{g l y}{ }^{2}$ was genotype-bylocation-by-crop year, $\sigma_{\varepsilon}{ }^{2}$ was error, $r$ was number of replications, $l$ was number of locations and $y$ was crop years respectively.

GGE biplot completed by "GGEBiplotGUI" package in $R$ software [60] was used to screen the stable soybean varieties with early flowering and late flowering in multienvironment. The general model of GGE biplot based on singular value decomposition (SVD) of environmentcentered or environment-standardized [61] could be written as:

$$
Y_{i j}-\mu-\beta_{j}=\lambda_{1} \xi_{i 1} \eta_{j 1}+\lambda_{2} \xi_{i 2} \eta_{j 2}+\varepsilon_{i j}
$$

where $Y_{i j}$ was the measured mean of $i^{\text {th }}$ genotype in $j^{\text {th }}$ environment; $\mu$ was the grand mean; $\beta_{j}$ was the main effect of $j^{\text {th }}$ environment; $\mu+\beta_{j}$ was the average trait over all genotypes in $j^{\text {th }}$ environment; $\lambda_{1}$ and $\lambda_{2}$ were the singular values for the first and second principal component (PC1 and PC2); $\xi_{i 1}$ and $\xi_{i 2}$ were eigenvectors of $i^{\text {th }}$ genotype for PC1 and PC2; $\eta_{j 1}$ and $\eta_{j 2}$ were eigenvectors of $j^{\text {th }}$ environment for PC1 and PC2; $\varepsilon_{i j}$ was the residual of the model associated with $i^{\text {th }}$ genotype in $j^{\text {th }}$ environment.

\section{Genotyping and quality control}

The double enzyme group comprising MseI and HaeIII (Thermo Fisher Scientific Inc., Waltham, MA, USA.) was used to digest the soybean genomic DNA that isolated from the fresh leaves of a single plant [62] into more than 50,000 sequencing tags, based on which, the sequencing libraries of each accession were constructed $[33,63]$. The Short Oligonucleotide Alignment Program 2 was used to map raw paired-end reads of the $45 \mathrm{bp}$ sequence read at both ends of the sequencing tags for each library, which was obtained using the barcode approach combined with the Illumina Genome Analyzer II (Illumina Inc., San Diego, CA, USA) onto the reference genome [64]. Approximately 58,000 high-quality SLAF tags were obtained after sequencing reads with the same genomic position of each accession. A total of 34,710 SNP loci with missing rate less than $10 \%$ and minor allele frequency (MAF) greater than 0.04 was used for GWAS.

\section{Linkage disequilibrium (LD), population structure and kinship analyses}

Pairwise LD between 34,710 SNP loci with missing rate less than $10 \%$ and MAF greater than 0.04 was estimated using squared allele frequency correlations $\left(\mathrm{r}^{2}\right)$ in TASSEL 5.0, the LD decay rate of the population was measured as the chromosomal distance when $\mathrm{r}^{2}$ dropped to half its maximum value $[38,65,66]$. The 34,710 SNP loci were also used to perform principal component analysis and calculate kinship matrixes by identity-by-state (IBS) method implemented in TASSEL 5.0 to infer population stratification and relatedness among individuals $[65,67]$.

\section{GWAS and candidate genes prediction}

The statistical mixed linear model (CMLM-PCA $+\mathrm{K}$ ) was used to perform GWAS using TASSEL 5.0 [65]. The equation for the CMLM-PCA $+\mathrm{K}$ analysis was expressed as:

$$
y=X \alpha+P \beta+Z \mathrm{u}+\mathrm{e}
$$

where $y$ was phenotype value; $\alpha$ was the vector of SNP effects; $\beta$ was vector of population structure effects based on PCA; $u$ was vector of kinship background effects; e was vector of residual effects; $X, P, Z$ were incidence matrix relating the individuals to fixed marker 
effects $\alpha$, fixed principal component (PC) effects $\beta$, random group effects $\mathrm{u}$, respectively.

Bonferroni test $P<0.05 / \mathrm{n}$ or $P<0.01 / \mathrm{n}(n=278)$, that was $-\log _{10} P>3.75$ or $-\log _{10} P>4.44$, was used to determine the significant or extremely significant SNP-trait associations. The R-based package snp.plotter was used to comb the LD block of the significant SNPs, the candidate genes associated with soybean flowering time within which were predicted using the SoyBase (https://www. soybase.org) and TAIR (https://www.arabidopsis.org/) databases.

\section{Supplementary information}

Supplementary information accompanies this paper at https://doi.org/10 1186/s12864-019-6324-7.

Additional file 1. General information and phenotypic data of 278 accessions used in this study.

Additional file 2. Statistical analysis of growth periods traits for 278 soybean varieties in six environments.

Additional file 3. Variance analysis (ANOVA) of 278 soybean varieties in six environments.

Additional file 4. The soybeans with stable below- or above-average flowering time.

Additional file5. The raw data and the corresponding accession numbers by SLAF-seq.

Additional file 6. SNPs distribution on each chromosome.

Additional file 7. Peak SNP associated with soybean growth periods in one environment by CMLM-PCA + K model.

Additional file 8. Peak SNPS associated with soybean growth periods in at least two environments by CMLM-PCA + K model.

Additional file 9. The 291 genes within the LD block $\left(r^{2}>0.5\right)$ of 37 significant SNPS.

Additional file 10: Figure S1. The normal score of standard normal random variable transformed from growth periods for 278 soybeans.

Additional file 11: Figure S2. Phenotypic correlation analysis of 278 soybean varieties in six environments.

Additional file 12: Figure S3. Manhattan and QQ plots of GWAS for soybean growth periods.

\section{Abbreviations}

CMLM: Compressed mixed linear model; GWAS: Genome-wide association study; LD: Linkage disequilibrium; MAF: Minor allele frequencies;

PCA: Principal component analysis; $R^{2}$ : Phenotypic variance; SLAF-seq: Specific locus amplified fragment sequencing; SNP: Single nucleotide polymorphisms

\section{Acknowledgements}

This study was conducted in the Key Laboratory of Soybean Biology of the Chinese Education Ministry, the Soybean Research \& Development Center (CARS) and the Key Laboratory of Northeastern Soybean Biology and Breeding/Genetics of the Chinese Agriculture Ministry.

\section{Authors' contributions}

$L Z, X Z, D M L$ and $W B L$ designed and supervised the research; MML and $Y L$ participated in the phenotypic data collection; $X \mathrm{~L}$, YHT and CJX performed phenotyping; $L Z, X Z, M M L, Y P H, X Y, J Z S, X M Z$ conducted the molecular experiments and the association analysis; and $L Z, W B L$ and MML wrote the manuscript. All authors read and approved the final manuscript.

\section{Funding}

This study was conducted in the Key Laboratory of Soybean Biology of Chinese Education Ministry, Key Laboratory of Biology, Genetics \& Breeding for Soybean in Northeast China, Ministry of Agriculture, Soybean Research \& Development Center, CARS, and financially supported by Key Special Project National Key Research \& Development Program 'seven crop breeding' (2016YFD0101005), National Natural Science Foundation of China (31771820), Chinese Key Projects of Soybean Transformation (2016ZX08004-005), The national project (CARS-04-PS04). None of the funding bodies participated in the design of the study, collection, analysis, and interpretation of data, or writing the manuscript.

\section{Availability of data and materials}

All data generated or analyzed during this study are included in this published article and its supplementary information files.

Ethics approval and consent to participate

The plant materials were collected from germplasms bank of the Key Laboratory of Soybean Biology of the Chinese Education Ministry. The collection and usage of samples followed the ethics of the People's Republic of China.

\section{Consent for publication}

Not applicable.

\section{Competing interests}

The authors declare that they have no competing interests.

Received: 20 July 2019 Accepted: 22 November 2019

Published online: 16 December 2019

\section{References}

1. Watanabe S, Harada K, Abe J. Genetic and molecular bases of photoperiod responses of flowering in soybean. Breed Sci. 2011;61(5):531-43.

2. Cober ER, Morrison MJ. Regulation of seed yield and agronomic characters by photoperiod sensitivity and growth habit genes in soybean. Theor Appl Genet. 2010;120(5):1005-12.

3. Zhang JP, Song QJ, Cregan PB, Nelson RL, Wang XZ, Wu JX, Jiang GL. Genome-wide association study for flowering time, maturity dates and plant height in early maturing soybean (Glycine max) germplasm. BMC Genomics. 2015;16(1):217. (https://link.springer.com/article/10.1186/s12864015-1441-4).

4. Bernard RL. Two major genes for time of flowering and maturity in soybeans. Crop Sci. 1971;11(2):242-4.

5. Bonato ER, Vello NA. E6, a dominant gene conditioning early flowering and maturity in soybeans. Genet Mol Biol. 1999;22(2):229-32.

6. Valéria CP, De ALA, RAdS K. Inheritance of a long juvenile period under short-day conditions in soybean. Genet Mol Biol. 2002;25(4):463-9.

7. Watanabe S, Hideshima R, Xia ZJ, Tsubokura Y, Sato S, Nakamoto Y, Yamanaka N, Takahashi R, Ishimoto M, Anai T, et al. Map-based cloning of the gene associated with the soybean maturity locus E3. Genetics. 2009; 182(4):1251-62.

8. Buzzell RI. Inheritance of a soybean flowering response to fluorescentdaylength conditions. Can J Genet Cytol. 1971;13(4):703-7.

9. Samanfar B, Molnar SJ, Charette M, Schoenrock A, Dehne F, Golshani A, Belzile F, Cober ER. Mapping and identification of a potential candidate gene for a novel maturity locus, E10, in soybean. Theor Appl Genet. 2017; 130(2):377-90.

10. Mcblain BA, Bernard RL. A new gene affecting the time of flowering and maturity in soybeans. J Hered. 1987:78(3):160-2.

11. Kong F, Nan H, Dong C, Ying L, Wu F, Wang J, Lu S, Yuan X, Abe J, Cober ER. A new dominant gene $E 9$ conditions early flowering and maturity in soybean. Crop Sci. 2014;54(6):2529-35.

12. Cober ER, Voldeng HD. A new soybean maturity and photoperiod-sensitivity locus linked to and. Crop Sci. 2001:41(3):698-701.

13. Cober ER, Molnar SJ, Charette M, Voldeng HD. A new locus for early maturity in soybean. Crop Sci. 2010:50(2):524-7.

14. Watanabe S, Harada K, Abe J. Genetic and molecular bases of photoperiod responses of flowering in soybean. Breed Sci. 2012:61(5):531-43.

15. Lu SJ, Zhao XH, Hu YL, Liu SL, Nan HY, Li XM, Fang C, Cao D, Shi XY, Kong $L P$, et al. Natural variation at the soybean J locus improves adaptation to the tropics and enhances yield. Nat Genet. 2017;49(5):773-9. 
16. Liu B, Kanazawa A, Matsumura H, Takahashi R, Harada K, Abe J. Genetic redundancy in soybean Photoresponses associated with duplication of the Phytochrome a gene. Genetics. 2008;180(2):995-1007.

17. Yue YL, Liu NX, Jiang BJ, Li M, Wang HJ, Jiang Z, Pan HT, Xia QJ, Ma QB, Han TF, et al. A single nucleotide deletion in J encoding GmELF3 confers long juvenility and is associated with adaption of tropic soybean. Mol Plant. 2017;10(4):656-8.

18. Wu F, Price BW, Haider W, Seufferheld G, Nelson R, Hanzawa Y. Functional and evolutionary characterization of the CONSTANS gene family in shortday photoperiodic flowering in soybean. PLoS One. 2014;9(1):e85754.

19. Na X, Jian B, Yao W, Wu C, Hou W, Jiang B, Bi Y, Han T. Cloning and functional analysis of the flowering gene GmSOC1-like, a putative SUPPRESSOR OF OVEREXPRESSION CO1/AGAMOUS-LIKE 20 (SOC1/AGL20) ortholog in soybean. Plant Cell Rep. 2013;32(8):1219-29.

20. Zhang Q, Li H, Li R, Hu R, Fan C, Chen F, Wang Z, Liu X, Fu Y, Lin C. Association of the circadian rhythmic expression of GmCRY1a with a latitudinal cline in photoperiodic flowering of soybean. Proc Natl Acad Sci U S A. 2008;105(52):21028-33.

21. Zhao K, Tung CW, Eizenga GC, Wright MH, Ali ML, Price AH, Norton GJ, Islam MR, Reynolds A, Mezey J, et al. Genome-wide association mapping reveals a rich genetic architecture of complex traits in Oryza sativa. Nat Commun. 2011;2:467. (https://www.nature.com/articles/ncomms146 7?message-global=remove).

22. Nordborg M, Weigel D. Next-generation genetics in plants. Nature. 2008; 456(7223):720-3.

23. Weng J, Xie C, Hao Z, Wang J, Liu C, Li M, Zhang D, Bai L, Zhang S, Li X. Genome-wide association study identifies candidate genes that affect plant height in Chinese elite maize (Zea mays L.) inbred lines. Plos One. 2011; 6(12):e29229.

24. Su J, Fan S, Li L, Wei H, Wang C, Wang H, Song M, Zhang C, Gu L, Zhao S, et al. Detection of favorable QTL alleles and candidate genes for lint percentage by GWAS in Chinese upland cotton. Front Plant Sci. 2016;7:1576.

25. Zhao X, Han YP, Li YH, Liu DY, Sun MM, Zhao Y, Lv CM, Li DM, Yang ZJ, Huang $L$, et al. Loci and candidate gene identification for resistance to Sclerotinia sclerotiorum in soybean (Glycine max L. Merr.) via association and linkage maps. Plant J. 2015;82(2):245-55.

26. Chen W, Yao J, Chu L, Yuan Z, Li Y, Zhang Y. Genetic mapping of the nulliplex-branch gene (gb_nb1) in cotton using next-generation sequencing. Theor Appl Genet. 2015;128(3):539-47.

27. Mao T, Li J, Wen Z, Wu T, Wu C, Shi S, Jiang B, Hou W, Li W, Song Q. Association mapping of loci controlling genetic and environmental interaction of soybean flowering time under various photo-thermal conditions. BMC Genomics. 2017;18(1):415.

28. Fang C, Ma Y, Wu S, Liu Z, Wang Z, Yang R, Hu G, Zhou Z, Yu H, Zhang M. Genome-wide association studies dissect the genetic networks underlying agronomical traits in soybean. Genome Biol. 2017;18(1):161.

29. Zhou L, Wang SB, Jian J, Geng QC, Wen J, Song Q, Wu Z, Li GJ, Liu YQ, Dunwell JM. Identification of domestication-related loci associated with flowering time and seed size in soybean with the RAD-seq genotyping method. Sci Rep. 2015;5:9350

30. Zhao X, Teng W, Li Y, Liu D, Cao G, Li D, Qiu L, Zheng H, Han Y, Li W. Loc and candidate genes conferring resistance to soybean cyst nematode HG type 2.5.7. Bmc Genomics. 2017;18(1):462.

31. Micheaux PLD, Drouilhet RM, Liquet BT. The R software : fundamentals of programming and statistical analysis. New York: Springer Publishing Company; 2013.

32. Song DWG. The study on the heritability and coefficient of hereditary variation of maize variety resources. J Hubei Agric Coll. 1999;19(3):212-4.

33. Han YP, Zhao X, Liu DY, Li YH, Lightfoot DA, Yang ZJ, Zhao L, Zhou G, Wang ZK, Huang $L$, et al. Domestication footprints anchor genomic regions of agronomic importance in soybeans. New Phytol. 2016;209(2):871-84.

34. Wen Z, Tan R, Yuan J, Bales C, Du W, Zhang S, Chilvers MI, Schmidt C, Song Q, Cregan PB. Genome-wide association mapping of quantitative resistance to sudden death syndrome in soybean. BMC Genomics. 2014;15(1):809.

35. Yu J, Gael P, Briggs WH, Irie VB, Masanori Y, Doebley JF, Mcmullen MD, Gaut BS, Nielsen DM, Holland JB. A unified mixed-model method for association mapping that accounts for multiple levels of relatedness. Nat Genet. 2006; 38(2):203-8.

36. Qu MN, Jiang BJ, Liu W, Mao TT, Ma LM, Lin KX, Han TF, University NA. New approaches to molecular breeding of soybean. J Agric Sci Technol. 2014; 16(3):8-13.
37. Flint-Garcia SA, Thornsberry JM, Buckler ES. Structure of linkage disequilibrium in plants. Annu Rev Plant Biol. 2003;54:357-74.

38. Huang XH, Wei XH, Sang T, Zhao QA, Feng Q, Zhao Y, Li CY, Zhu CR, Lu TT, Zhang ZW, et al. Genome-wide association studies of 14 agronomic traits in rice landraces. Nat Genet. 2010;42(11):961-967. (https://www.nature.com/ articles/ng.695).

39. Morris GP, Ramu P, Deshpande SP, Hash CT, Shah T, Upadhyaya HD, RieraLizarazu O, Brown PJ, Acharya CB, Mitchell SE, et al. Population genomic and genome-wide association studies of agroclimatic traits in sorghum. Proc Natl Acad Sci U S A. 2013;110(2):453-8.

40. Yan JB, Shah T, Warburton ML, Buckler ES, Mcmullen MD, Jonathan C. Genetic characterization and linkage disequilibrium estimation of a global maize collection using SNP markers. PLoS One. 2009;4(12):e8451.

41. Yong W, Kang C, Xu Z, Tan K, Zhu Z. Gene control of flowering time in higher plants. Chin Sci Bull. 2000;45(18):1633-42.

42. Srikanth A, Schmid M. Regulation of flowering time: all roads lead to Rome. Cell Mol Life Sci. 2011;68(12):2013-37.

43. Tiwari SB, Shen Y, Chang HC, Hou Y, Harris A, Ma SF, McPartland M, Hymus GJ, Adam L, Marion C. The flowering time regulator CONSTANS is recruited to the FLOWERING LOCUS T promoter via a unique cis-element. New Phytol. 2010;187(1):57-66.

44. Song YH, Smith RW, To BJ, Millar AJ, Imaizumi T. FKF1 conveys timing information for CONSTANS stabilization in photoperiodic flowering. Science. 2012;336(6084):1045-9.

45. Torti S, Fornara F, Vincent C, Andrés F, Nordström K, Göbel U, Knoll D, Schoof $\mathrm{H}$, Coupland $\mathrm{G}$. Analysis of the Arabidopsis shoot meristem transcriptome during floral transition identifies distinct regulatory patterns and a leucine-rich repeat protein that promotes flowering. Plant Cell. 2012; 24(2):444-62.

46. Geraldo N, Baurle I, Kidou S, Hu X, Dean C. FRIGIDA delays flowering in Arabidopsis via a cotranscriptional mechanism involving direct interaction with the nuclear cap-binding complex. Plant Physiol. 2009;150(3):1611-8.

47. Wei L, II-Pyung A, Yuese N, Chan-Ho P, Lirong Z, Whitehill JGA, Haibin L, Qingzhen Z, Bo D, Qi X. The U-box/ARM E3 ligase PUB13 regulates cell death, defense, and flowering time in Arabidopsis. Plant Physiol. 2012;159(1): 239-50.

48. Li J, Yang X, Wang Y, Li X, Gao Z, Pei M, Chen Z, Qu L-J, Gu H. Two groups of MYB transcription factors share a motif which enhances trans-activation activity. Biochem Biophys Res Commun. 2006;341(4):1155-63.

49. Lai AG, Doherty CJ, Mueller-Roeber B, Kay SA, Schippers JH, Dijkwel PP. CIRCADIAN CLOCK-ASSOCIATED 1 regulates ROS homeostasis and oxidative stress responses. Proc Natl Acad Sci. 2012;109(42):17129-34.

50. Li G, Siddiqui H, Teng Y, Lin R, Wan X-Y, Li J, Lau O-S, Ouyang X, Dai M, Wan J. Coordinated transcriptional regulation underlying the circadian clock in Arabidopsis. Nat Cell Biol. 2011;13(5):616.

51. Lu SX, Webb CJ, Knowles SM, Kim SH, Wang Z, Tobin EM. CCA1 and ELF3 interact in the control of hypocotyl length and flowering time in Arabidopsis. Plant Physiol. 2012;158(2):1079-88.

52. Duong S, Vonapartis E, Li C-Y, Patel S, Gazzarrini S. The E3 ligase ABI3INTERACTING PROTEIN2 negatively regulates FUSCA3 and plays a role in cotyledon development in Arabidopsis thaliana. J Exp Bot. 2017;68(7):1555-67.

53. Han Y, Zhao X, Cao G, Wang Y, Li Y, Liu D, Teng W, Zhang Z, Li D, Qiu L, et al. Genetic characteristics of soybean resistance to $\mathrm{HG}$ type 0 and $\mathrm{HG}$ type 1.2.3.5.7 of the cyst nematode analyzed by genome-wide association mapping. BMC Genomics. 2015;16:598.

54. Maag JLV. gganatogram: An R package for modular visualisation of anatograms and tissues based on ggplot2. F1000Res. 2018;7:1576.

55. Kahle D, Wickham H. ggmap: spatial visualization with ggplot2. R J. 2013; 5(1):144-61.

56. Fehr WR, Caviness CE. Stages of soybean development. In: Special Report 80. vol. 80. Ames: lowa State University of Science and Technology; 1977. p. 11.

57. Field A. Discovering statistics using IBM SPSS statistics. 5th ed. Thousand Oaks: University of Sussex SAGE Publications Ltd; 2013.

58. Tang QY, Zhang CX. Data processing system (DPS) software with experimental design, statistical analysis and data mining developed for use in entomological research. Insect Sci. 2013;20(2):254-60.

59. Jamoza JE, Owuoche J, Kiplagat O, Opile W. Broad-sense heritability estimation and correlation among sugarcane (Saccharum spp. hybrids) yield and some agronomic traits in western Kenya. Int J Agric Policy Res. 2014; 2(1):16-25. 
60. Frutos $E$, Galindo MP, Leiva V. An interactive biplot implementation in R for modeling genotype-by-environment interaction. Stoch Env Res Risk A. 2014; 28(7):1629-41.

61. Yan WK. Optimal use of biplots in analysis of multi-location variety test data. Acta Agron Sin. 2010;36(11):1805-19.

62. Wu XL, Ren CW, Joshi T, Vuong T, Xu D, Nguyen HT. SNP discovery by highthroughput sequencing in soybean. BMC Genomics. 2010;11(1):469-0. (https://link.springer.com/article/10.1186/1471-2164-11-469).

63. Sun XW, Liu DY, Zhang XF, Li WB, Liu H, Hong WG, Jiang CB, Guan N, Ma CX, Zeng HP, et al. SLAF-seq: An Efficient Method of Large-Scale De Novo SNP Discovery and Genotyping Using High-Throughput Sequencing. PLoS One. 2013;8(3):e58700. (https://journals.plos.org/plosone/article?id=10.1371/ journal.pone.0058700).

64. Li R, Yu C, Li Y, Lam TW, Yiu SM, Kristiansen K, Wang J. SOAP2: an improved ultrafast tool for short read alignment. Bioinformatics. 2009:25(15):1966-7.

65. Bradbury PJ, Zhang Z, Kroon DE, Casstevens TM, Ramdoss Y, Buckler ES. TASSEL: software for association mapping of complex traits in diverse samples. Bioinformatics. 2007;23(19):2633-5.

66. Remington DL, Thornsberry JM, Matsuoka Y, Wilson LM, Whitt SR, Doebley J, Kresovich S, Goodman MM, Buckler ES. Structure of linkage disequilibrium and phenotypic associations in the maize genome. Proc Natl Acad Sci U S A. 2001:98(20):11479-84.

67. Endelman JB, Jannink JL. Shrinkage estimation of the realized relationship matrix. G3 (Bethesda). 2012;2(11):1405-13.

\section{Publisher's Note}

Springer Nature remains neutral with regard to jurisdictional claims in published maps and institutional affiliations.

Ready to submit your research? Choose BMC and benefit from:

- fast, convenient online submission

- thorough peer review by experienced researchers in your field

- rapid publication on acceptance

- support for research data, including large and complex data types

- gold Open Access which fosters wider collaboration and increased citations

- maximum visibility for your research: over $100 \mathrm{M}$ website views per year

At $\mathrm{BMC}$, research is always in progress.

Learn more biomedcentral.com/submissions 